\title{
Single Mouse Oocyte Encapsulated in Medium-in-Oil Microdroplets by Using a Polydimethylsiloxane Microfluidic Device
}

\author{
Hsien-Hua Shen ${ }^{1}$, Hsiu-Yun Tsai ${ }^{2}$ and Da-Jeng Yao ${ }^{1,3, *}$ \\ ${ }^{1}$ Institute of Nanoengineering and Microsystems, National Tsing Hua University, Hsinchu, Taiwan \\ ${ }^{2}$ Institute of Biomedical Engineering, National Tsing Hua University, Hsinchu, Taiwan \\ ${ }^{3}$ Department of Power Mechanical Engineering, National Tsing Hua University, Hsinchu, Taiwan
}

(Received June 10 2013; accepted October 2, 2013)

Key words: microfluidic device, microdroplet generation, polystyrene beads, and mouse oocyte

A polydimethylsiloxane (PDMS) flow-focusing microfluidic device was used to generate micrometer medium-in-oil microdroplets, and a single mouse oocyte was encapsulated in each dispersed microdroplet for in vitro fertilization (IVF) research. The droplet-based microfluidics provides a dynamic and noncontinuous culture condition for mimicking the in vivo cell development environment. Cell culture in monodispersed microdroplets requires less medium volumes, reduces cell culture cost, prevents contamination risk, and minimizes evaporation. This PDMS microfluidic channel contains two inlets for continuous oil injection and medium injection with oocytes. The oocyte in the culture medium was embedded into microdroplets by shear force and dispersed in the oil phase. The volume of microdroplets can be controlled by adjusting the flow rates of both oil and medium inlets. After microdroplets containing a single oocyte were generated stably into the outlet of the microfluidic channel, the microdroplets could be collected in a tube for further biomedical applications.

\section{Introduction}

With structure design and sensor integration, micro-electromechanical systems (MEMS)-based devices have been widely used in chemical or biomedical research such as in chemical separation, ${ }^{(1)}$ chemical detection, ${ }^{(2,3)}$ protein crystallization, ${ }^{(4)}$ single-nucleotide polymorphism (SNP) detection, ${ }^{(5)}$ DNA extraction, ${ }^{(6,7)}$ polymerase chain reaction, ${ }^{(8)}$ cell culture, ${ }^{(9)}$ cell separation, ${ }^{(10-12)}$ and drug delivery systems. ${ }^{(13)}$ The advantages of using microfluidics are to reduce reagent sample consumption and to shorten the processing time. However, the traditional continuous microfluidic system also has some problems such as low reagent mixing efficiency and sample

${ }^{*}$ Corresponding author: e-mail: djyao@mx.nthu.edu.tw 
contamination. To solve these problems, droplet-based microfluidics ${ }^{(9,14-16)}$ was used in several biomedical applications such as single-cell hybridoma screening, ${ }^{(17)}$ digital analysis of bacterial samples, ${ }^{(18)}$ and immunoassay-based single-cell analysis and sorting. ${ }^{(19)}$ Some research groups have used cross-flow or T-junction ${ }^{(20)}$ structures for water-in-oil microdroplet dispersion, and others used co-flow microfluidics ${ }^{(21)}$ for liquid emulsification.

In vitro fertilization (IVF) has been a very important reproductive technique for infertility in recent years. The oocyte from a female ovary is fertilized with sperm cells outside the body. Then, the fertilized embryo is cultivated in an oil-covered tube or a culture dish. In the traditional IVF process, repeated washing and pipetting might introduce the risk of cell loss and condition change, such as changes in $\mathrm{pH}$, gas concentration, nutrient concentration, and temperature. Recent IVF studies tend to use a continuous polydimethylsiloxane (PDMS) microfluidic device for biomedical sample processing including oocyte pretreatment, sperm sorting, and further embryo culturing. ${ }^{(12,22,23)}$ However, it is hard to manipulate multiple oocytes in a continuous channel, and contamination is also a serious problem in biomedical experiments. The research goal is to generate microdroplets encapsulating a single mouse oocyte for further IVF. A flow-focusing microfluidic device ${ }^{(24,25)}$ was applied for high-throughput monodispersed medium-in-oil microdroplet generation, shown in Fig. 1. The main structures are a PDMS microfluidic channel with two inlets for oil and medium and one outlet for the output of generated microdroplets. Culture oil is continuously pushed into the oil inlet by a syringe pump, and the medium with mouse oocytes is pushed into the medium inlet by another syringe pump, which is called the push-push controlling method. Continuous oil streams provide shear force near the nozzle of the microfluidic channel to break the continuous medium phase into dispersed microdroplets with oocytes. The volume of microdroplets can be controlled by changing the flow rates of syringe pumps.

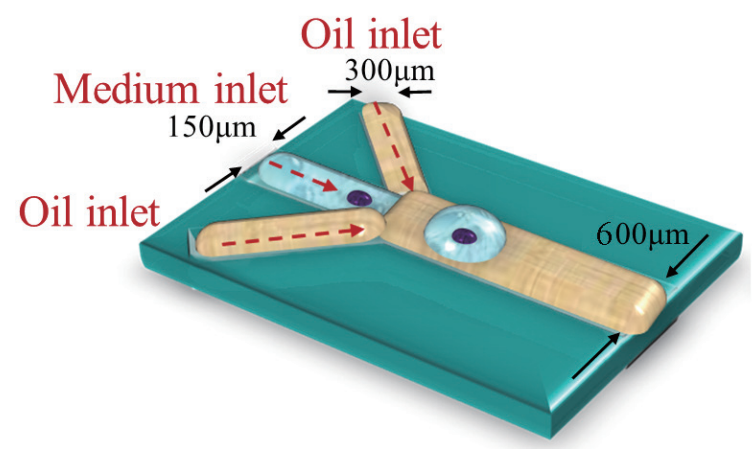

Fig. 1. (Color online) Schematic of PDMS microfluidic device. Dispersed microdroplets can be formed by shear force. The height of the channel is $195 \mu \mathrm{m}$, and the widths of the oil and medium inlets are 150 and $300 \mu \mathrm{m}$, respectively. 


\section{Materials and Methods}

\subsection{PDMS microfluidic device fabrication process}

The PDMS microfluidic channel is widely used in biomedical research, and its good biocompatibility has been proved. The PDMS microfluidic channel was fabricated through photolithography and polymer fabrication techniques as shown on Fig. 2. A Si substrate was firstly spin-coated and patterned by using SU-8 3050 negative photoresist to make a master. The SU-8 film thickness was about $195 \mu \mathrm{m}$, which would define the height of a microfluidic channel. The main structure of microfluidic devices was made by a PDMS replica molding process. After the PDMS was cured at $85{ }^{\circ} \mathrm{C}$ for $1 \mathrm{~h}$ in an oven, the microfluidic channel was peeled off from the master. Then, inlets and the outlet were made using standard 3-mm-diameter punching tools. Finally, the PDMS structure was bonded on glass by oxygen plasma surface treatment to finish the microfluidic device fabrication process.

\subsection{Surface modification of microfluidic channel}

After the PDMS structure was bonded on a glass substrate by oxygen plasma surface treatment, the surface of the microfluidic channel would become hydrophilic. Medium would just spread out on the hydrophilic wall and directly flow into the outlet without

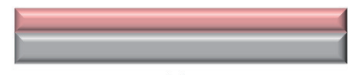

(a)

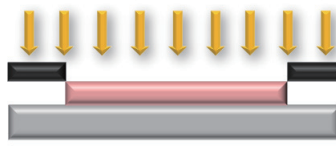

(b)

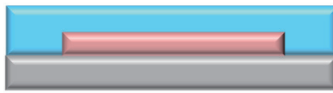

(c)

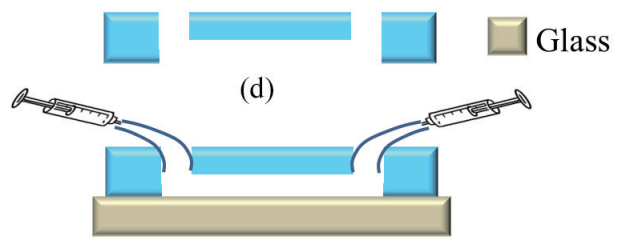

(e)

Si wafer

Mask

SU8

PDMS

lass

(d)

Fig. 2. (Color online) Fabrication of PDMS microfluidic device. (a) SU8 negative photoresist was spin-coated on Si wafer as the master. (b) Photoresist was exposed and developed. (c) PDMS curing and peeling off from the master. (d) Inlets and outlet definition using 3-mm-diameter punching tools. (e) PDMS structure was bonded on the glass substrate, and the channel inlets were connected by plastic tubes into a syringe pump. 
creating medium microdroplets in the oil phase. To avoid this phenomenon, surface treatment on the channel wall is necessary for further microfluidic device fabrication. Aquape ${ }^{\circledR}$ is a better hydrophobic material for channel surface treatment than Teflon ${ }^{\circledR}$ or Cytop $^{\circledR}$. Aquapel ${ }^{\circledR}$ is a fluorinated compound developed by Pittsburgh Plate Glass (PPG) Company. This fluoropolymer is able to bind on a glass surface to make the surface hydrophobic. Before loading medium and oil for experiments, $100 \mu$ l of Aquapel ${ }^{\circledR}$ was loaded and flowed into the microfluidic channel for surface modification.

\subsection{Oocyte pretreatment and culture medium preparation}

Female mice were treated with 5 IU of pregnantmare serum gonadotrophin (PMSG) by intraperitoneal injection to stimulate the development of ovarian follicles. After around 42 to $48 \mathrm{~h}, 5 \mathrm{IU}$ of human chorionic gonadotrophin (HCG) was injected to obtain more oocytes. Finally, the mice were euthanized after 10-13 h, and the oocytes were obtained from oviducts. They were then placed on a dish containing human tubal fluid (HTF) culture medium.

HTF culture medium produced by Irvine Scientific ${ }^{\circledR}$ is a synthetic culture medium for embryo development and gamete manipulation. It is bicarbonate-based and is designed for use in a $\mathrm{CO}_{2}$ incubator. Mineral culture oil produced by $\mathrm{OVOIL}^{\mathrm{TM}}$ is a sterile and light paraffin oil, which is usually used for covering the HTF medium during in vitro cell culture procedures.

\subsection{Chip packaging and microfluidic system setup}

After the fabrication process, the PDMS microfluidic device was bonded with glass substrate by $\mathrm{O}_{2}$ plasma treatment. Figure 3(a) shows the top view of the microfluidic device with two inlets and one outlet. Medium with oocytes was pumped into the middle channel through the medium inlet, and oil was pumped into the channel through the oil inlet and then separated into two streams. The top and bottom oil streams provided shear force near the nozzle for medium microdroplet generation.

The full size of the chip is about $5 \times 2.6 \mathrm{~cm}^{2}$. The widths of the oil and medium phase channels are 300 and $150 \mu \mathrm{m}$, respectively, and the outlet channel is $600 \mu \mathrm{m}$. During the experiment, the oil inlet was connected to a flexible small-bore transparent tubing of $3 \mathrm{~mm}$ diameter and sealed by multipart adhesives to prevent liquid leakage. The medium inlet was connected to a tube-shaped connector and syringe for HTF culture medium loading and injection. Finally, the syringes were connected to syringe pumps to complete the microfluidic system setup, as shown in Fig. 3(b). Images of the channel were observed using an in-line metalloscope and the videos were recorded with a standard CCD camera.

\section{Results}

\subsection{Controllable volume of microdroplets}

First of all, to create microdroplets using this design, water instead of medium was loaded and pushed into the medium inlet by a syringe pump for testing, and then a continuous oil phase was also pushed into two oil inlets by another syringe pump. After 


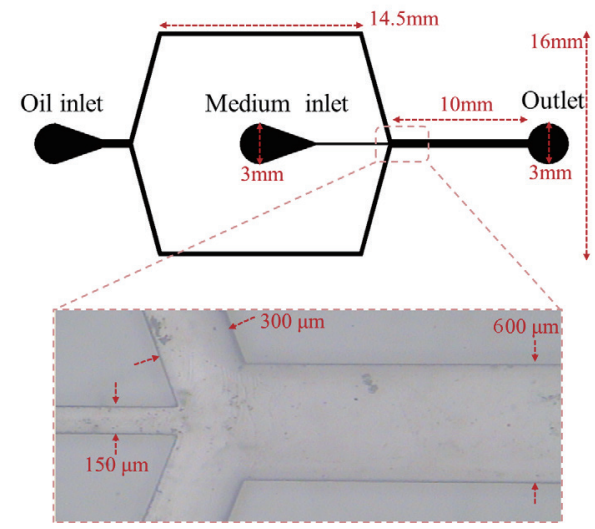

(a)

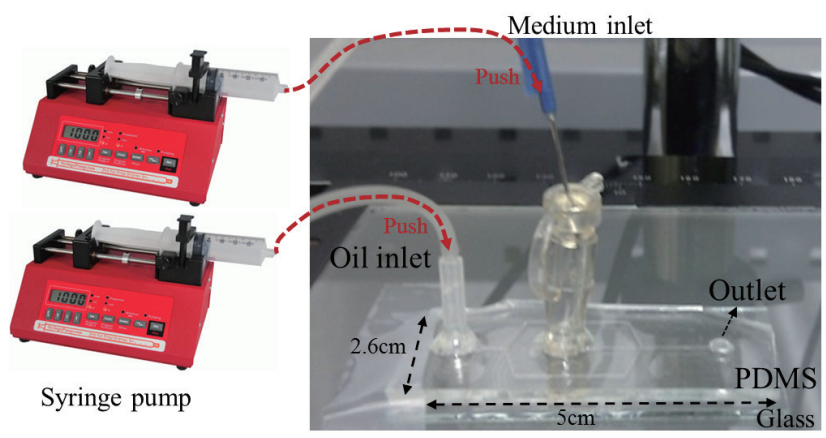

(b)

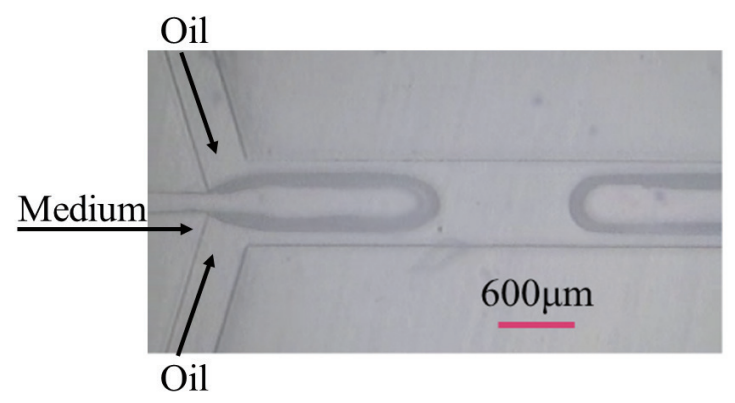

(c)

Fig. 3. (Color online) Schematic of PDMS microfluidic device. (a) Pattern of this PDMS microfluidic device and top-view image of the flow-focusing microfluidic channel. (b) Experimental setup. The samples were injected into the channel using syringe pumps. (c) Dispersed microdroplets were generated in the flow-focusing microfluidic channel.

successfully obtaining the water microdroplets, the HTF medium without oocyte was used to replace the medium in the inlet for the HTF microdroplet generation process. Shear force generated by continuous oil streams near the nozzle broke the continuous medium phase into monodispersed microdroplets, as shown on Fig. 3(c).

The volume of microdroplets could be controlled by changing the flow rates of oil and medium. Figure 4(a) shows the relationship between volume of microdroplets and flow rate of the oil phase inlet when the flow rate of DI water or medium phase is fixed. By using both DI water and HTF medium for the medium inlet, they all showed that the volumes of microdroplets created by a slow flow rate $(50 \mu \mathrm{l} / \mathrm{h})$ are smaller than those created by a fast flow rate $(500 \mu \mathrm{l} / \mathrm{h})$ under the same flow rate of the oil phase. However, the volume variation of microdroplets would be small and stable if a slower flow rate is used at the medium inlet. We also found out that the volume of a DI water microdroplet 


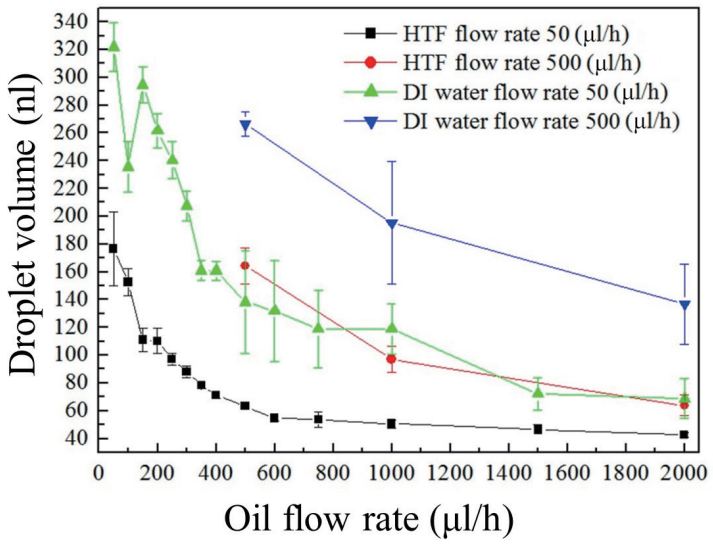

(a)

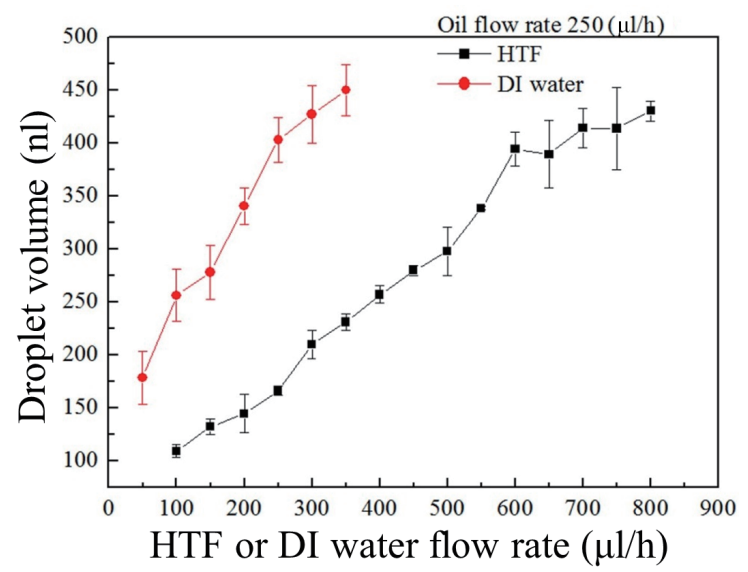

(b)

Fig. 4. (Color online) Microdroplet volume analysis. (a) Relationship between microdroplet volume and flow rate of oil phase when the flow rate of DI water or medium phase is fixed. (b) Relationship between microdroplet volume and flow rate of DI water or medium phase when the flow rate of oil phase is set at $250 \mu \mathrm{l} / \mathrm{h}$.

was about twice larger than that of an HTF medium microdroplet generated at the same flow rate.

In IVF research, the minimum volume of a medium microdroplet for oocyte culture is about $100 \mathrm{nl}$, so we must determine how to generate stably $100 \mathrm{nl}$ microdroplets by controlling the flow rates of all the inlets. When the flow rate of the HTF medium was set at $50 \mu \mathrm{l} / \mathrm{h}$, the volume of a dispersed microdroplet decreased from 180 to $40 \mathrm{nl}$ when the flow rate of the oil phase was increased from 50 to $2000 \mu \mathrm{l} / \mathrm{h}$. Thus, the flow rate of the oil phase was selected to be at $250 \mu \mathrm{l} / \mathrm{h}$ to obtain $100 \mathrm{nl}$ HTF microdroplets. Figure 4(b) shows that the volume of a dispersed HTF microdroplet increased from 110 to 425 $\mathrm{nl}$ when the flow rate of the HTF medium phase was increased from 50 to $2000 \mu \mathrm{l} / \mathrm{h}$.

\subsection{Single polystyrene bead or oocyte embedded in each microdroplet}

From the results above, the flow rates of the HTF medium and oil phase were set at 50 and $200 \mu \mathrm{l} / \mathrm{h}$, respectively, to generate around $100 \mathrm{nl}$ dispersed medium-in-oil HTF microdroplets. To encapsulate oocytes into HTF microdroplets, polystyrene (PS) beads were used instead of oocytes for device testing because mouse oocytes are valuable and hard to obtain. Figure 5 shows the formation of HTF microdroplet containing a single PS bead. The first HTF microdroplet with a PS bead was generated by steps shown in Figs. 5(a)-5(c), and another one was generated by steps shown in Figs. 5(d)-5(f). In the serial microdroplet generation process, a few HTF microdroplets would contain more than one PS beads because of the latter's high concentration.

The next step is to use oocytes instead of PS beads in this research. The concentration of oocytes in the medium was relatively low in comparison with that of 
the PS beads, which may create the possibility of some HTF microdroplets containing no oocyte. Figure 6 shows a single oocyte embedded in an HTF microdroplet when the flow rates of the HTF medium and oil were set at 50 and $200 \mu \mathrm{l} / \mathrm{h}$, respectively.

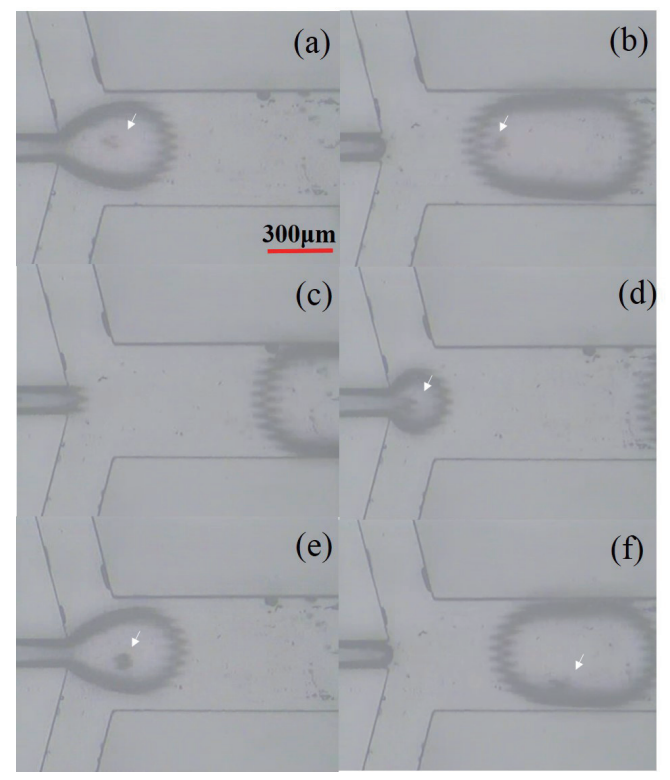

Fig. 5. (Color online) Sequential images of generation of HTF microdroplets containing a single PS bead. (a)-(c) A microdroplet is formed with a PS bead inside. (d)-(f) Another microdroplet is formed with a PS bead inside. The flow rates of HTF and oil were set at 50 and $200 \mu \mathrm{l} / \mathrm{h}$, respectively.

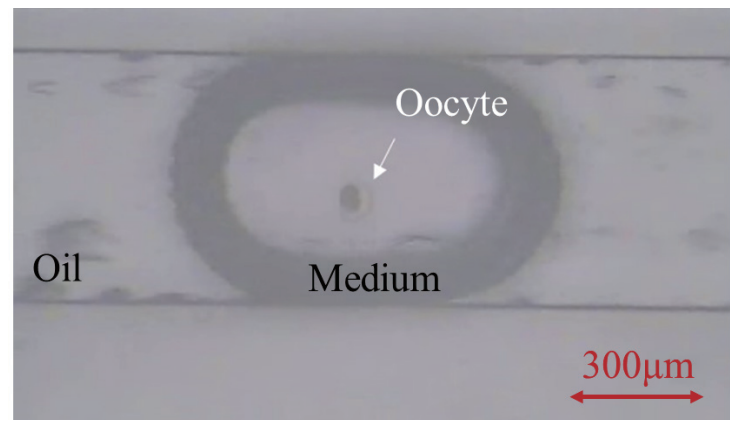

Fig. 6. (Color online) A single oocyte embedded in an HTF microdroplet when medium and oil flow rates were set at 50 and $200 \mu \mathrm{l} / \mathrm{h}$, respectively. 


\section{Conclusions}

We successfully used PDMS flow-focusing microfluidics for medium-in-oil microdroplet generation. Nanoliter-sized HTF microdroplets with a single mouse oocyte are formed in the oil phase by shear force. The volume of microdroplets can be controlled by adjusting the flow rates of two syringe pumps. When the flow rates of the medium and oil are set at 50 and $200 \mu \mathrm{l} / \mathrm{h}$, respectively, $100 \mathrm{nl}$ HTF microdroplets are generated by the push-push controlling method, which is the minimum volume for further IVF cell culture or observation. In the future, this medium-in-oil microdroplet generation microfluidic device will be used for IVF research for decreasing the probability of cell loss and providing a stable cell culture condition.

\section{Acknowledgements}

The authors would like to thank Prof. Fan-Gang Tseng of the Department of Engineering and System Science at National Tsing Hua University, Hsinchu, Taiwan for providing fabrication facilities. This work was partially supported by the National Science Council, Taiwan, under grant no. NSC 101-2221-E-007-009-MY3.

\section{References}

1 J. S. Edgar, G. Milne, Y. Zhao, C. P. Pabbati, D. S. Lim and D. T. Chiu: Angew. Chem. Int. Ed. 48 (2009) 2719

2 K. T. Tang, D. J. Yao, C. M. Yang, H. C. Hao, J. S. Chao, C. H. Li and P. S. Gu: Proc. Olfaction and Electronic Nose 1137 (2009) 86.

3 P. H. Ku, C. Y. Hsiao, M. J. Chen, T. H. Lin, Y. T. Li, S. C. Liu, K. T. Tang, D. J. Yao and C. M. Yang: Langmuir 28 (2012) 11639.

4 L. Li and R. F. Ismagilov: Annu. Rev. Biophys. 39 (2010) 139.

5 H. H. Shen, T. Y. Su, Y. J. Liu and D. J. Yao: Proc. Sens. Mater. 25 (2013) 643.

6 Y. C. Chung, M. S. Jan, Y. C. Lin, J. H. Lin, W. C. Cheng and C. Y. Fan: Lab Chip 4 (2004) 141.

7 Y. Zhang, S. Park, S. Yang and T. H. Wang: Biomed. Microdevices 12 (2010) 1043.

8 P. Kumaresan, C. J. Yang, S. A. Cronier, R. G. Blazej and R. A. Mathies: Anal. Chem. 80 (2008) 3522.

9 J. Clausell-Tormos, D. Lieber, J. C. Baret, A. El-Harrak, O. J. Miller, L. Frenz, J. Blouwolff, K. J. Humphry, S. Koster, H. Duan, C. Holtze, D. A. Weitz, A. D. Griffiths and C. A. Merten: Chem. Biol. 15 (2008) 427.

10 C. L. Chen, K. C. Chen, Y. C. Pan, T. P. Lee, L. C. Hsiung, C. M. Lin, C. Y. Chen, C. H. Lin, B. L. Chiang and A. M. Wo: Lab Chip 11 (2011) 474.

11 J. Takagi, M. Yamada, M. Yasuda and M. Seki: Lab Chip 5 (2005) 778.

12 H. Y. Huang, T. L. Wu, H. R. Huang, C. J. Li, H. T. Fu, Y. K. Soong, M. Y. Lee and D. J. Yao: Proc. JALA 19 (2014) 91.

13 B. Ziaie, A. Baldi, M. Lei, Y. Gu and R. A. Siegel: Adv. Drug Delivery Rev. 56 (2004) 145.

14 R. Seemann, M. Brinkmann, T. Pfohl and S. Herminghaus: Rep. Prog. Phys. 75 (2012) 016601 . 
15 T. M. Tran, F. Lan, C. S. Thompson and A. R. Abate: J. Phys. D: Appl. Phys. 46 (2013) 114004.

16 Y. T. Chen, W. C. Chang, W. F. Fang, S. C. Ting, D. J. Yao and J. T. Yang: Microfluid. Nanofluid. 13 (2012) 239.

17 B. El Debs, R. Utharala, I. V. Balyasnikova, A. D. Griffiths and C. A. Merten: Proc. Natl. Acad. Sci. USA 109 (2012) 11570.

18 M. Najah, A. D. Griffiths and M. Ryckelynck: Anal. Chem. 84 (2012) 1202.

19 L. Mazutis, J. Gilbert, W. L. Ung, D. A. Weitz, A. D. Griffiths and J. A. Heyman: Nat. Protoc. 8 (2013) 870.

20 P. Garstecki, M. J. Fuerstman, H. A. Stone and G. M. Whitesides: Lab Chip 6 (2006) 437.

21 A. Perro, C. L. Nicolet, J. Angly, S. B. Lecommandoux, J.-F. O. Le Meins and A. Colin: Langmuir 27 (2010) 9034.

22 J. E. Swain, D. Lai, S. Takayama and G. D. Smith: Lab Chip 13 (2013) 1213.

23 M. D. C. Lopez-Garcia, R. L. Monson, K. Haubert, M. B. Wheeler and D. J. Beebe: Biomed. Microdevices 10 (2008) 709.

24 Z. Nie, M. Seo, S. Xu, P. Lewis, M. Mok, E. Kumacheva, G. Whitesides, P. Garstecki and H. Stone: Microfluid. Nanofluid. 5 (2008) 585.

25 M. Mulligan and J. Rothstein: Microfluid. Nanofluid. 13 (2012) 65.

\section{About the Authors}

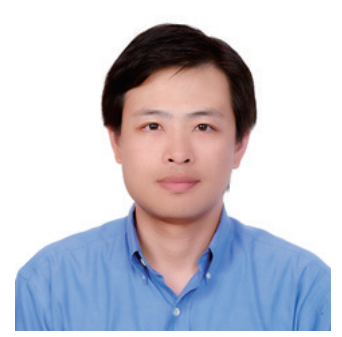

Da-Jeng Yao is a professor and director of the Institute of NanoEngineering and MicroSystems (NEMS), National Tsing Hua University, Taiwan. He was born in Taipei, Taiwan in 1969. He earned his Ph.D. from the Department of Mechanical and Aerospace Engineering, University of California at Los Angeles (UCLA) in 2001. His research objective is to combine strong backgrounds in MEMS and thermal fluidics for microscience research. To build a multidisciplinary research team for the development of interdisciplinary technologies within his research scope is his ambition at National Tsing Hua University. He has published more than 50 journal papers in top journals in this research field, including Biosensors and Bioelectronics, Nanotechnology, Carbon, Journal of Micromechanics and Microengineering, and Lap on a Chip. He won the best patent and best paper award from the Industrial Technology Research Institute (ITRI), Wu-Da-Yu Memorial Award (Young Investigator) from the National Science Council in 2009, Shen-Yin award in 2010, and ASME fellowship in 2013.

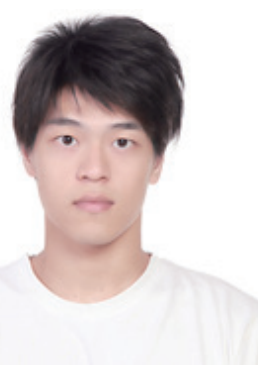

Hsien-Hua Shen is a Ph.D. student of the Institute of NanoEngineering and MicroSystems at National Tsing Hua University, Taiwan. Mr. Shen has a B.S. in Life Sciences and an M.S. in NanoEngineering and MicroSystems, both from National Tsing Hua University, Taiwan (R.O.C.). He, under the Bio-Medical Thermo-Fluidics Lab, is focusing on biomedical applications of the EWOD digital microfluidic system. 


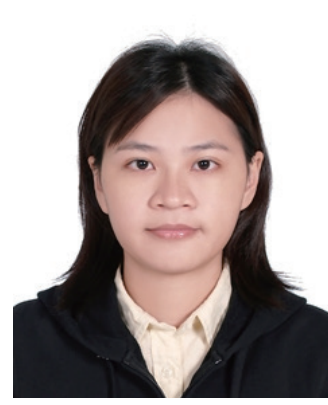

Hsiu-Yun Tsai is a master's student of the Institute of Biomedical Engineering at National Tsing Hua University. Tsai received her B.S. degree in Biological Science \& Technology at I-Shou University in Taiwan (R.O.C.). She is currently a graduate student in the Bio-Medical Thermo-Fluidics Lab. Her research focuses on droplet generation and particle separation using PDMS microfluidic devices. 